\title{
Dynamic linkages between stock markets: the effects of crises and globalization
}

\author{
Małgorzata Doman · Ryszard Doman
}

Received: 25 April 2011 / Accepted: 2 June 2013 / Published online: 13 July 2013

(C) The Author(s) 2013. This article is published with open access at Springerlink.com

\begin{abstract}
This paper investigates changes in the dynamics of linkages between selected national stock markets during the period 1995-2009. The analysis focuses on the possible effects of globalization and differences between crisis and non-crisis periods. We model the dynamics of dependencies between the series of daily returns on selected stock indices over different time periods, and compare strength of the linkages. Our tools are dynamic copula models and a formal sequential testing procedure based on the model confidence set methodology. We consider two types of dependencies: regular dependence measured by means of the conditional Spearman's rho, and dependencies in extremes quantified by the conditional tail dependence coefficients. The main result consists of a collection of rankings created for the considered subperiods, which show how the mean level of strength of the dependencies have been changing in time. The rankings obtained for Spearman's rho and tail dependencies differ, which allows us to distinguish between the results of crises and the effect of globalization.
\end{abstract}

Keywords Globalization · Crisis · Stock index · Co-movement · Spearman's rho · Tail dependence $\cdot$ Copula $\cdot$ Model confidence set

JEL Classifications $\mathrm{G} 15 \cdot \mathrm{G} 01 \cdot \mathrm{C} 58 \cdot \mathrm{C} 32$

\footnotetext{
M. Doman

Department of Applied Mathematics, Poznań University of Economics, Al. Niepodległości 10, 61-875 Poznań, Poland

e-mail: malgorzata.doman@ue.poznan.pl

R. Doman $(\bowtie)$

Laboratory of Financial Econometrics, Faculty of Mathematics and Computer Science,

Adam Mickiewicz University in Poznań, Umultowska 87, 61-614 Poznań, Poland

e-mail: rydoman@amu.edu.pl
} 


\section{Introduction}

In the paper, we look for the effects of globalization and crises on the pattern of linkages in global stock market by modeling the conditional dependencies between the series of daily returns on selected stock indices during various periods. The dependencies in each period are modeled using a regime-switching copula model. Two types of dependencies are considered: regular dependence measured by means of the conditional Spearman's rho, and dependencies in extremes quantified by the conditional tail dependence coefficients. The main result is a collection of rankings created for the considered subperiods, which show how the average level of strength of the dependencies have been changing in time. The rankings obtained for Spearman's rho and tail dependencies differ, which allows us to distinguish between the results of crises and the effect of globalization.

The main motivation for the analysis originates from a common opinion explaining why the web of inter-market linkages become more dense and stronger. It states that this is because financial capital can now move very quickly from one part of the world into another and any information reaches investors in all the world almost in the same time when it appears.

The increase of dependencies influences world economies and markets creating new investment possibilities but, at the same time, new risks. Because of the importance of this process for risk management and macroeconomic policies, the attempts to describe and explain it form a significant part of research in many areas of finance. Among the first empirical papers dealing with this subject, the papers by Levy and Sarnat (1970) and Solnik (1974) analyzing correlations across national markets and international portfolio diversification should be mentioned. Many empirical research papers concerning the problem of market linkages arose in the 1990s (for instance, Ammer and Mei (1996), Campbell and Hamao (1992), and Pindick and Rotemberg (1993)). The sequence of financial crises that occurred at the end of the 20th century gave impact to intensive work on the subject. For instance, in 2003 a special issue of Journal of Economics and Business edited by Bailey and Choi (2003) was dedicated to the international market linkages analysis focused on the degree of financial and economic integration. The crisis of 2007-2009 further intensified the attempts to describe and explain the mechanism of the dependencies in global financial market.

In the wide literature on the subject many theoretical papers exist that address the problem of the strengthening of market dependencies and the related problem of propagation of shocks that arise in one country and spread to some other ones. The papers consider diverse approaches and use variety of methodologies. The broad set of theories concerning the topic can be generally divided into two groups: crisiscontingent and non-crisis-contingent theories (Forbes and Rigobon 2002; Gallo and Velucchi 2009). The former explain whether cross-market linkages increase after a shock, whereas the latter assume that transmission mechanisms are always the same and that cross-market linkages do not increase after a shock. In this paper, we are not concerned with studying the related phenomena of contagion, interdependence or spillover (see, for example, Gallo and Otranto 2008) but just investigate whether and to what extent the dynamics of the linkages changes. 
The question about the best way to describe the dependencies between different markets is still open. For a long time the most common method applied to measure the linkages was correlation (e.g., Hamao et al. 1990; Karolyi and Stulz 1996). Next, multivariate GARCH models were used to describe the time-varying conditional correlations of global equity returns (see e.g. Capiello et al. 2006). There also exist papers using cointegration as a tool to examine the long-run equilibrium relationships between national capital markets (Bachman et al. 1996; Gérard et al. 2003). Another useful tool applied recently to describe inter-market dependencies are copulas (Jondeau and Rockinger 2006; Bartram et al. 2007; Rodriguez 2007; Chen and Poon 2007; Okimoto 2008; Chollete et al. 2009; Markwat et al. 2009).

In the presented research we try to answer the following questions: Can the globalization effect be measured by means of econometric tools? Do the results of measurement of the strength of market linkages indicate an increasing tendency? Did the market linkages dynamics change during the crises periods? Did tail dependencies, especially lower tail dependence, become stronger when a crisis occurred?

To answer these questions we need an econometric measure of dependencies that is robust to specific properties of financial return series. As it was mentioned before, classic studies on interdependencies between financial returns commonly use simple, dynamic or exceedance, correlation. However, outside the world of elliptical distributions using the linear correlation is not appropriate because of possibility of quite misleading inference (Embrechts et al. 2002; McNeil et al. 2005). To avoid such problems we use copulas as a tool to describe the pattern of connections. These functions have the property to join any kind of margins into a multivariate distribution function. An advantage of the copula approach is that it allows to separate the dependence structure from the marginal distributions. By means of copulas it is also possible to model tail behavior in the joint distribution, and different kinds of asymmetry, what is of great importance in our investigation. However, in order to capture changes in the conditional dependence structure, the copula that describes it must be time varying. Time variation in the conditional copula was introduced in 2002 by Patton (2004, 2006), and it had the form of an ARMA-type process involving the dependence parameter of the copula and a function of return innovations. Another, alternative approach, which is also applied in the present paper, may be that the model includes a number of regimes that are switched according to a Markov chain, and different copulas prevail at particular regimes (Rodriguez 2007; Markwat et al. 2009; Garcia and Tsafack 2011).

The analysis presented in this paper focuses on the linkages between stock indices representing 11 countries and is based on the series of percentage logarithmic daily returns. It is performed for 16 pairs of stock indices, 10 of which include the S\&P 500, and 6 the DAX. The dataset under scrutiny embrace quotations from January 3, 1995 to December 11, 2009. The dynamics of market linkages is described using Spearman's rho and the coefficients of tail dependence. After estimation of the models and computing the sequences of the considered conditional dependence measures we compare the strength of linkages in different periods using the Model Confidence Set methodology (Hansen et al. 2011) and create a set of rankings. This allows us to draw some conclusions about the impact of the 
globalization process and financial crises on the pattern of dependencies in global financial market.

\section{Regime-switching copula models}

Modeling the dependencies between financial returns is a difficult task because of special properties of these series. Typical return series usually exhibit conditional heteroskedasticity, different types of asymmetries, and structural breaks, which strongly influence estimation results for models of the dependence structure. Moreover, the dynamics of dependencies can significantly change in time. For example, it is well documented in many studies that dependence between returns on different assets is usually stronger in bear markets than in bull markets (Ang and Bekaert 2002; Ang and Chen 2002; Patton 2004). This example of asymmetric dependence in financial markets is of great importance for portfolio choice and risk management. The main problem connected with this phenomenon is, however, that from the theoretical point of view, the mentioned asymmetry cannot be produced by a statistical model for the returns that assumes an elliptical multivariate conditional distribution, and thus applying the linear correlation is not justified. An alternative concept that allows for modeling the dependence in general situation is copula. Roughly speaking, a bivariate copula is a mapping $C:[0,1] \times[01] \rightarrow[0,1]$ from the unit square into the unit interval which is a distribution function with standard uniform marginal distributions.

Assume that $(X, Y)$ is a 2-dimensional random vector with joint distribution $H$ and marginal distributions $F$ and $G$. Then, by a theorem by Sklar (1959), $H$ can be written as

$$
H(x, y)=C(F(x), G(y)) .
$$

If $F$ and $G$ are continuous then the function $C$ is uniquely given by the formula

$$
C(u, v)=H\left(F^{-1}(u), G^{-1}(v)\right),
$$

for $u, v \in[0,1]$, where $F^{-1}(u)=\inf \{x: F(x) \geq u\}$. In this case, $C$ is called the copula of $H$ or of $(X, Y)$. Since the marginals and the dependence structure can be separated, it makes sense to interpret $C$ as the dependence structure of the vector $(X, Y)$. We refer to Patton (2009) and references therein for an overview of financial time series applications of copulas. There one can also find more information about advantages and limitations of copula-based modeling.

The simplest copula is defined by $C^{\Pi}(u, v)=u v$ and it corresponds to independence of marginal distributions.

In the empirical part of this paper we will also use the Gaussian and Joe-Clayton copulas. They are defined as follows:

$$
C_{\rho}^{\text {Gauss }}(u, v)=\Phi_{\rho}\left(\Phi^{-1}(u), \Phi^{-1}(v)\right),
$$

$$
C_{\kappa, \gamma}^{\text {Joe-Clayton }}(u, v)=1-\left(1-\left(\left[1-(1-u)^{\kappa}\right]^{-\gamma}+\left[1-(1-v)^{\kappa}\right]^{-\gamma}-1\right)^{-1 / \gamma}\right)^{1 / \kappa}
$$

Here in (3), $\Phi_{\rho}$ denotes the distribution of a 2-dimensional standardized normal vector with the linear correlation coefficient $\rho$, and $\Phi$ stands for the standard normal 
distribution function. The parameters in the Joe-Clayton copula (4) are assumed to satisfy the conditions: $\kappa \geq 1, \gamma>0$. For $\kappa=1$, the Joe-Clayton copula becomes the Clayton copula $C_{\gamma}^{\text {Clayton }}$. In the limit case $\gamma=0$, the Clayton copula approaches the independence copula $C$ (Nelsen 2006).

The density associated to an absolutely continuous copula $C$ is a function $c$ defined by

$$
c(u, v)=\frac{\partial^{2} C(u, v)}{\partial u \partial v} .
$$

For an absolutely continuous random vector, the copula density $c$ is related to its joint density function $h$ by the following canonical representation:

$$
h(x, y)=c(F(x), G(y)) f(x) g(y),
$$

where $F$ and $G$ are the marginal distributions, and $f$ and $g$ are the marginal density functions.

In the case of nonelliptical distributions, measures of dependence that are more appropriate than the linear correlation coefficient are provided by two important copula-based tools known as Kendall's tau and Spearman's rho (Embrechts et al. 2002). Since the dynamics of Spearman's rho is exploited in this paper, we recall a suitable definition. If $(X, Y)$ is a random vector with marginal distribution functions $F$ and $G$, then Spearman's rho for $(X, Y)$ can be defined as

$$
\rho_{S}(X, Y)=\rho(F(X), G(Y))
$$

where $\rho$ denotes the usual Pearson correlation. For continuous marginal distributions, Spearman's rho, $\rho_{S}(X, Y)$, depends only on the copula $C$ linking $X$ and $Y$, and, in particular, it is given by the formula

$$
\rho_{S}(X, Y)=\rho_{C}=12 \iint_{[0,1]^{2}} C(u, v) d u d v-3
$$

(Nelsen 2006). It follows from (8) that if a copula $C$ is a mixture of copulas $C_{1}$ and $C_{2}: C=\alpha C_{1}+(1-\alpha) C_{2}, 0 \leq \alpha \leq 1$, then

$$
\rho_{C}=\alpha \rho_{C_{1}}+(1-\alpha) \rho_{C_{2}} \text {. }
$$

For the Gaussian copula $C_{\rho}^{\text {Gauss }}$, Spearman's rho equals $\frac{6}{\pi} \arcsin \frac{1}{2} \rho$, and for the Joe-Clayton copula it can be computed numerically using (8).

A very important concept connected with copula, relevant to dependence in extreme values, is tail dependence. If $X$ and $Y$ are random variables with distribution functions $F$ and $G$ then the coefficient of upper tail dependence is defined as follows

$$
\lambda_{U}=\lim _{q \rightarrow 1^{-}} P\left(Y>G^{-1}(q) \mid X>F^{-1}(q)\right),
$$

provided a limit $\lambda_{U} \in[0,1]$ exists. Analogously, the coefficient of lower tail dependence is defined as

$$
\lambda_{L}=\lim _{q \rightarrow 0^{+}} P\left(Y \leq G^{-1}(q) \mid X \leq F^{-1}(q)\right),
$$

provided that a limit $\lambda_{L} \in[0,1]$ exists. If $\lambda_{U} \in(0,1]\left(\lambda_{L} \in(0,1]\right)$, then $X$ and $Y$ are said to exhibit upper (lower) tail dependence. Upper (lower) tail dependence 
quantifies the likelihood to observe a large (low) value of $Y$ given a large (low) value of $X$. The coefficients of tail dependence depend only on the copula $C$ of $X$ and $Y$ :

$$
\begin{aligned}
& \lambda_{L}=\lim _{q \rightarrow 0^{+}} \frac{C(q, q)}{q}, \\
& \lambda_{U}=\lim _{q \rightarrow 0^{+}} \frac{\hat{C}(q, q)}{q},
\end{aligned}
$$

where $\hat{C}(u, v)=u+v-1+C(1-u, 1-v)$. For the Gaussian copula it holds $\lambda_{U}=\lambda_{L}=0$ (see Embrechts et al. 2002), meaning asymptotic independence in the tails. In the Joe-Clayton copula, $\lambda_{U}=2-2^{1 / \kappa}$ and $\lambda_{L}=2^{-1 / \gamma}$ for $\gamma>0$ (Patton 2006). Thus both upper and lower dependence can be nonzero, and, moreover, they can change freely of each other.

Introduced by Patton (2004), the notion of conditional copula allows to apply copulas to modeling the joint distribution of $\mathbf{r}_{t}$ conditional on information set $\Omega_{t-1}$, where $\mathbf{r}_{t}=\left(r_{1, t}, r_{2, t}\right)$ is a bivariate vector of financial returns. In this paper we consider the following general conditional copula model

$$
\begin{aligned}
r_{1, t} \mid \Omega_{t-1} & \sim F_{t}(\cdot), \quad r_{2, t} \mid \Omega_{t-1} \sim G_{t}(\cdot), \\
\mathbf{r}_{t} \mid \Omega_{t-1} & \sim C_{t}\left(F_{t}(\cdot), G_{t}(\cdot) \mid \Omega_{t-1}\right),
\end{aligned}
$$

where the set $\Omega_{t}$ includes the up to time $t$ information on the returns on both considered financial assets, and $C_{t}$ is the conditional copula linking the marginal conditional distributions. Further, we assume that

$$
\begin{gathered}
\mathbf{r}_{t}=\boldsymbol{\mu}_{t}+\mathbf{y}_{t}, \quad \boldsymbol{\mu}_{t}=E\left(\mathbf{r}_{t} \mid \Omega_{t-1}\right), \\
y_{i, t}=\sigma_{i, t} \varepsilon_{i, t}, \quad \sigma_{i, t}^{2}=\operatorname{var}\left(r_{i, t} \mid \Omega_{t-1}\right), \\
\varepsilon_{i, t} \sim \text { iid Skew_t }\left(0,1, \xi_{i}, \eta_{i}\right),
\end{gathered}
$$

where $S_{k e w} t(0,1, \xi, \eta)$ denotes the standardized skewed Student $t$ distribution with $\eta>2$ degrees of freedom, and skewness coefficient $\xi>0$ (Lambert and Laurent 2001). To the marginal return series $r_{i, t}, i=1,2$, we fit ARMA-GARCH models with skewed Student's $t$ distributions for the 1-dimensional innovations.

When modeling the joint conditional distribution, the evolution of the conditional copula $C_{t}$ has to be specified. Usually (Patton 2004, 2006), the functional form of the conditional copula is fixed, but its parameters evolve through time. In this paper, however, an alternative approach is applied. Similarly to Garcia and Tsafack (2011), we assume that there are regimes where a fixed copula prevails, and they switch according to some homogeneous Markov chain.

In a Markov-switching copula model (MSC model) we use for modeling the conditional dependence between financial returns, the joint conditional distribution has the following form

$$
\mathbf{r}_{t} \mid \Omega_{t-1} \sim C_{S_{t}}\left(F_{t}(\cdot), G_{t}(\cdot) \mid \Omega_{t-1}\right),
$$

where $S_{t}$ is a homogeneous Markov chain with state space $\{1,2\}$. Hence, the parameters of the applied MSC model are the parameters of the univariate models for the marginal distributions (ARMA-GARCH with the standardized skewed Student's $t$ 
distributions for the innovations), the parameters of the copulas $C_{1}$ and $C_{2}$, and the transition probabilities

$$
p_{11}=P\left(S_{t}=1 \mid S_{t-1}=1\right), \quad p_{22}=P\left(S_{t}=2 \mid S_{t-1}=2\right)
$$

of the Markov chain.

The parameters of the MSC model were estimated by the maximum likelihood method. The main by-product of the estimation are the predicted probabilities $P\left(S_{t}=j \mid \Omega_{t-1}\right), j=1,2$. They are calculated by means of Hamilton's filter (Hamilton 1994):

$$
\begin{gathered}
P\left(S_{t}=j \mid \Omega_{t-1}\right)=\sum_{i=1}^{2} p_{i j} P\left(S_{t-1}=i \mid \Omega_{t-1}\right), \\
P\left(S_{t}=j \mid \Omega_{t}\right)=\frac{c_{j}\left(\mathbf{u}_{t} \mid S_{t}=j, \Omega_{t-1}\right) P\left(S_{t}=j \mid \Omega_{t-1}\right)}{\sum_{i=1}^{2} c_{i}\left(\mathbf{u}_{t} \mid S_{t}=i, \Omega_{t-1}\right) P\left(S_{t}=i \mid \Omega_{t-1}\right)},
\end{gathered}
$$

where $p_{12}=P\left(S_{t}=2 \mid S_{t-1}=1\right)=1-p_{11}, p_{21}=P\left(S_{t}=1 \mid S_{t-1}=2\right)=$ $1-p_{22}, \mathbf{u}_{t}=\left(u_{1, t}, u_{2, t}\right)$,

$$
u_{1, t}=F_{t}\left(r_{1, t}\right), u_{2, t}=G_{t}\left(r_{2, t}\right),
$$

and $c_{j}\left(\cdot \mid S_{t}=j, \Omega_{t-1}\right)$ is the density of the conditional copula coupling the conditional marginal distributions in regime $j$. By the arguments of Hamilton (1994) and (6), the maximized log-likelihood function is of the form

$$
\begin{aligned}
L= & \sum_{t=1}^{T} \ln \left(\sum_{j=1}^{2} c_{j}\left(\mathbf{u}_{t} \mid S_{t}=j, \Omega_{t-1} ; \theta\right) P\left(S_{t}=j \mid \Omega_{t-1} ; \theta\right)\right) \\
& +\sum_{t=1}^{T} \ln \left(f_{t}\left(r_{1, t} \mid \Omega_{t-1} ; \theta_{1}\right)\right)+\sum_{t=1}^{T} \ln \left(g_{t}\left(r_{2, t} \mid \Omega_{t-1} ; \theta_{2}\right)\right),
\end{aligned}
$$

where $f_{t}$ and $g_{t}$ are the density functions corresponding to $F_{t}$ and $G_{t}$, fitted using ARMA-GARCH models.

\section{The model confidence set}

The Model Confidence Set (MCS) methodology was originally introduced by Hansen et al. (2003) in order to improve choosing the best volatility forecasting models from a larger set. The objective of this approach is to determine the set $\mathcal{M}^{*}$ that consists of the best models from some collection $\mathcal{M}^{0}$. Given a significance level $\alpha$, the MCS procedure produces a subset $\hat{\mathcal{M}}_{1-\alpha}^{*}$ of $\mathcal{M}^{0}$ that contains $\mathcal{M}^{*}$ with confidence level $1-\alpha$. Thus the MCS resembles a confidence interval for a parameter. In fact, $\mathcal{M}^{0}$ can be a finite set of objects indexed by $i=1, \ldots, m_{0}$ and evaluated in terms of a loss function that assigns to the object $i$ in period $t$ the loss $L_{i, t}, t=1, \ldots, n$. When one defines the relative performance variables as $d_{i j, t} \equiv L_{i, t}-L_{j, t}$ for $i, j \in \mathcal{M}^{0}$ 
and assumes that the mean $\mu_{i j} \equiv E\left(d_{i j, t}\right)$ is finite and does not depend on $t$, then the set of superior objects is defined by

$$
\mathcal{M}^{*} \equiv\left\{i \in \mathcal{M}^{0}: \mu_{i j} \leq 0 \text { for all } j \in \mathcal{M}^{0}\right\} .
$$

In order to determine $\mathcal{M}^{*}$, one tests the hypotheses of the form

$$
H_{0, \mathcal{M}}: \mu_{i j}=0 \text { for all } i, j \in \mathcal{M},
$$

where $\mathcal{M} \subset \mathcal{M}^{0}$. The alternative hypothesis, $\mu_{i j} \neq 0$ for some $i, j \in \mathcal{M}$, is denoted by $H_{A, \mathcal{M}}$. The MCS procedure is based on an equivalence test $\delta_{\mathcal{M}}$ that tests the hypothesis $H_{0, \mathcal{M}}$ for any $\mathcal{M} \subset \mathcal{M}^{0}$, and an elimination rule $e_{\mathcal{M}}$ that identifies the object of $\mathcal{M}$ that is to be removed from $\mathcal{M}$ when $H_{0, \mathcal{M}}$ is rejected. The MCS algorithm proposed by Hansen et al. (2003) is as follows:

Step 0: $\quad$ Set $\mathcal{M}=\mathcal{M}^{0}$.

Step 1: Test $H_{0, \mathcal{M}}$ using $\delta_{\mathcal{M}}$ at significance level $\alpha$.

Step 2: If $H_{0, \mathcal{M}}$ is not rejected define, $\hat{\mathcal{M}}_{1-\alpha}^{*}=\mathcal{M}$, otherwise use $e_{\mathcal{M}}$ to eliminate an object from $\mathcal{M}$ and repeat the procedure from Step 1.

It is proved in Hansen et al. (2011) that under some standard requirements for the equivalence test and the elimination rule, it holds that

$$
\begin{gathered}
\lim \inf _{n \rightarrow \infty} P\left(\mathcal{M}^{*} \subset \hat{\mathcal{M}}_{1-\alpha}^{*}\right) \geq 1-\alpha, \\
\lim _{n \rightarrow \infty} P\left(i \in \hat{\mathcal{M}}_{1-\alpha}^{*}\right)=0 \text { for all } i \notin \mathcal{M}^{*}, \\
\lim _{n \rightarrow \infty} P\left(\mathcal{M}^{*}=\hat{\mathcal{M}}_{1-\alpha}^{*}\right)=1 \text { if } \mathcal{M}^{*} \text { is a singleton. }
\end{gathered}
$$

If $P_{H_{0}, \mathcal{M}_{i}}$ denotes the $p$-value associated with the null hypothesis $H_{0, \mathcal{M}_{i}}$ (with convention that $\left.P_{H_{0}, \mathcal{M}_{m_{0}}}=1\right)$ then the MCS $p$-value for model $e_{\mathcal{M}_{j}} \in \mathcal{M}^{0}$ is defined as $\hat{p}_{e_{\mathcal{M}}} \equiv \max _{i \leq j} P_{H_{0}, \mathcal{M}_{i}}$.

It is shown in Hansen et al. (2011) that $H_{0, \mathcal{M}}$ can be tested using traditional quadratic-form statistics or multiple $t$-statistics. The latter approach has an advantage that it simplifies the construction of an elimination rule that satisfies the notion of coherency formulated in Hansen et al. (2011). We focus on the $t$-statistic $T_{R, \mathcal{M}}$ because we apply it in this paper. It is defined as

$$
T_{R, \mathcal{M}}=\max _{i, j \in \mathcal{M}}\left|t_{i j}\right|,
$$

where

$$
t_{i j}=\frac{\bar{d}_{i j}}{\sqrt{\operatorname{vâr}\left(\bar{d}_{i j}\right)}} \text { for } i, j \in \mathcal{M} \text {, }
$$

and $\bar{d}_{i j}=n^{-1} \sum_{t=1}^{n} d_{i j, t}$. The asymptotic distribution of the statistic $T_{R, \mathcal{M}}$ is nonstandard but it can be estimated with bootstrap methods. The bootstrap implementation of the MCS procedure involving this statistic is described in Appendix B in the paper by Hansen et al. (2003). For generating $B$ resamples the block bootstrap 
is used. The estimated bootstrap distribution of $T_{R, \mathcal{M}}$, under the null hypothesis, is given by the empirical distribution of

$$
T_{b, R}^{*}=\max _{i, j \in M} \frac{\left|\bar{d}_{b, i j}^{*}-\bar{d}_{i j}\right|}{\sqrt{\operatorname{vâr}\left(\bar{d}_{i j}\right)}},
$$

where $\bar{d}_{b, i j}^{*}=\frac{1}{n} \sum_{t=1}^{n} d_{i j, \tau_{b_{t}}}$ is the bootstrap resample average, and vâr $\left(\bar{d}_{i j}\right)=$ $\frac{1}{B} \sum_{b=1}^{B}\left(\bar{d}_{b, i j}^{*}-\bar{d}_{i j}\right)^{2}$. The $p$-value of $H_{0, \mathcal{M}}$ is given by

$$
P_{H_{0, \mathcal{M}}}=\frac{1}{B} \sum_{b=1}^{B} 1\left\{T_{b, R}^{*}>T_{R, \mathcal{M}}\right\} .
$$

It should be mentioned here that even though the MCS methodology was originally designed to select the best volatility forecasting models it can also be applied in any situation where one want to compare the means of two or more populations. We use the MCS procedure to establish rankings of subperiods with respect to the strength of linkages between the investigated stock markets calculated by the discussed dependence measures.

\section{The data}

The analysis is performed for 16 pairs of stock market indices. We consider five European indices (FTSE, DAX, CAC 40, the Russian RTS, and the Polish WIG20), four Asian (the NIKKEI 225, the South Korean KOSPI, the Singaporean STI, and the Indian BSE 30), the Brazilian BOVESPA, and the S\&P 500. In fact, we analyze only the dependencies between the S\&P 500 and all the other indices, and between the DAX and the FTSE, WIG20, RTS, NIKKEI, BSE 30 and KOSPI. The quotation series were obtained from the service Stooq. The period under scrutiny is from January 3, 1995 to December 11, 2009.

Since the patterns of non-trading days in national stock markets differ, for the purpose of modeling the dependencies, the dates of observations for the considered pairs of indices were checked and observations not corresponding to ones in the other index quotation series were removed. As the result, the number of observations changes from 3404 to 3732 , depending on the pair of indices. The time series under scrutiny are percentage logarithmic daily returns calculated by the formula

$$
r_{t}=100\left(\ln P_{t}-\ln P_{t-1}\right),
$$

where $P_{t}$ denotes the closing index value on day $t$.

Since we use international daily data, an issue of non-synchronous trading arises. None of the existing attempts to tackle this difficulty is fully satisfactory. In particular, using weekly data only seemingly solves the problem and, moreover, discards important information. For this reason, we adopt here the following approach. Prior to modeling, we compared by means of Pearson's correlation coefficient the strength of concurrent and one day lagged dependence between the components of pairs of 
Table 1 Summary statistics for daily log returns (in \%) for the period from January 2, 1995 to December 11,2009

\begin{tabular}{lccccrr}
\hline Index & Mean & Maximum & Minimum & Stand. Dev. & Skewness & Kurtosis \\
\hline BOVESPA & 0.0783 & 28.832 & -17.208 & 2.4278 & 0.4414 & 14.916 \\
BSE 30 & 0.0414 & 15.99 & -17.184 & 1.7992 & -0.4047 & 10.4106 \\
CAC 40 & 0.0190 & 10.595 & -9.4715 & 1.5051 & -0.0101 & 7.7121 \\
DAX & 0.0276 & 10.797 & -9.791 & 1.5877 & -0.0635 & 10.9230 \\
FTSE & 0.0146 & 9.3843 & -9.2656 & 1.2418 & -0.0939 & 9.0418 \\
KOSPI & 0.0137 & 11.284 & -16.115 & 2.0693 & -0.2841 & 7.5669 \\
NIKKEI & -0.0187 & 13.235 & -12.111 & 1.6046 & -0.2246 & 8.4993 \\
RTS & 0.0768 & 22.723 & -21.199 & 2.9858 & -0.2151 & 10.6200 \\
S\&P 500 & 0.0238 & 10.957 & -9.4695 & 1.289 & -0.1783 & 10.9195 \\
STI & 0.0060 & 12.874 & -15.517 & 1.4538 & -0.2677 & 12.6767 \\
WIG20 & 0.0302 & 13.709 & -14.161 & 1.9544 & -0.1548 & 6.7066 \\
\hline
\end{tabular}

the investigated return series. The presented results are obtained for those lags for which the correlation was the strongest. Consequently, we use one day lagged S\&P 500 returns in links with the indices WIG20, RTS, NIKKEI, STI and KOSPI.

Summary statistics for the analyzed return series are presented in Table 1 . They are calculated for the return series coupled with the S\&P 500 data, and the values for the S\&P 500 returns come from the series that was coupled with the FTSE.

\section{Empirical analysis}

In this paper, the changes in the strength of dependence are investigated in the following way. Given the total time period covered by our sample, we form a cascade of partitions of it into 2, 4, 8, and 15 intervals of equal length. For each partition, we try to fit the MSC models describing the dynamics of linkages between the S\&P 500 or DAX and the other stock indices in subperiods constituting the partition. In order to choose the best fitting models, we employ various copulas and apply information criteria, and likelihood ratio tests for the comparison of nested models (e.g. involving $C_{\gamma}^{\text {Clayton }}$ and $C_{\kappa, \gamma}^{\text {Joe-Clayton }}$, or $C \prod$ and $C_{\rho}^{\text {Gauss }}$ ). Using the estimated MSC copula models, we compute predicted values for Spearman's rho and tail dependence coefficients. Here, by the predicted value for Spearman's rho at time $t$ we mean the value $\rho_{t}$ calculated as

$$
\rho_{t}=\rho(1) P\left(S_{t}=1 \mid \Omega_{t-1}\right)+\rho(2) P\left(S_{t}=2 \mid \Omega_{t-1}\right),
$$

where $\rho(j)$ is Spearman's rho for a copula applicable in regime $j$. The predicted values of the lower and upper tail dependence coefficients are defined analogously. It follows from (8) that the $\rho_{t}$ is Spearman's rho for the mixture of copulas governing 


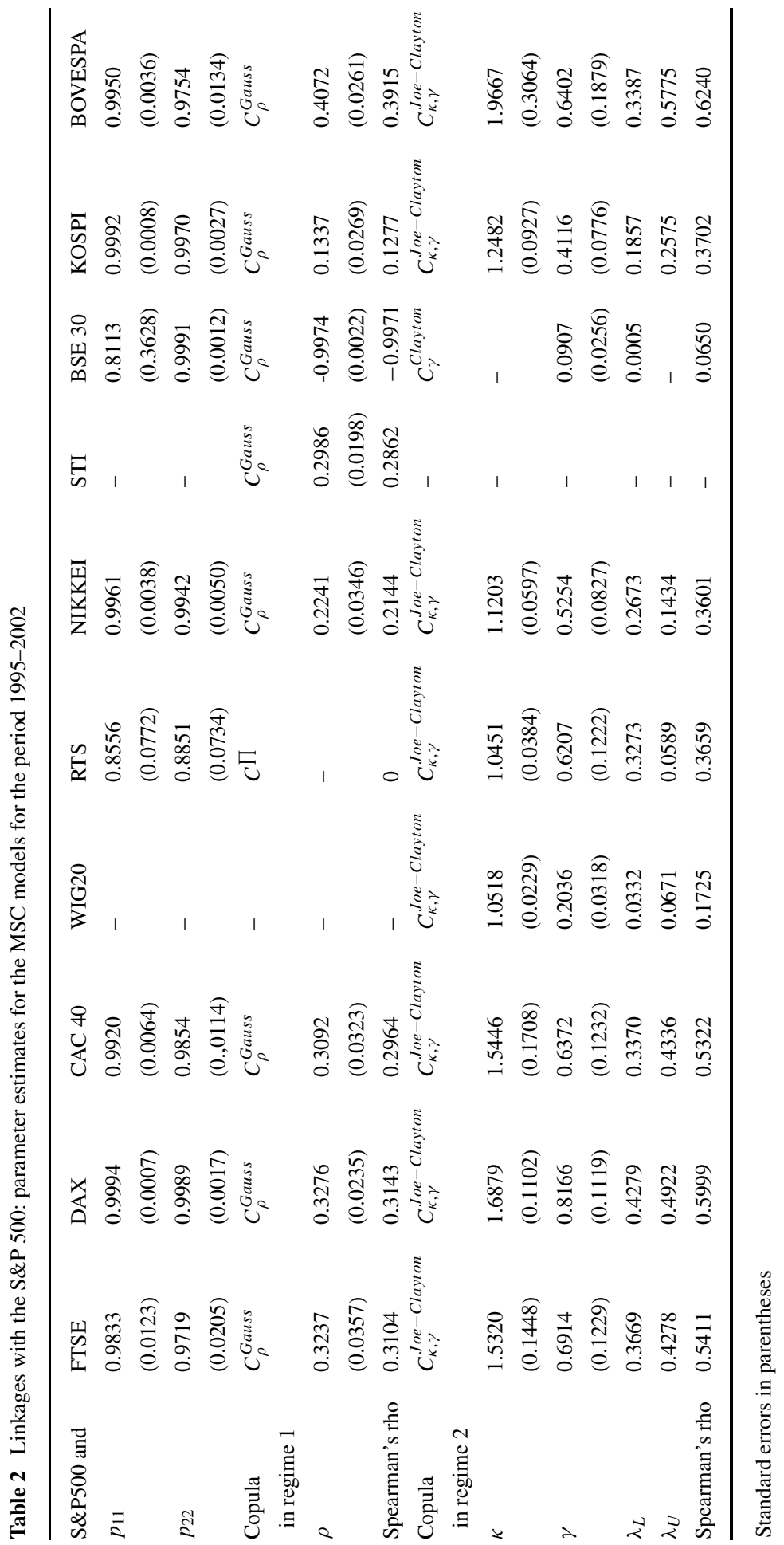




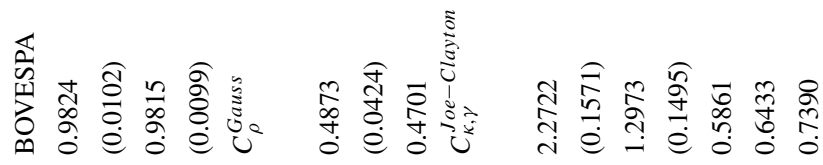

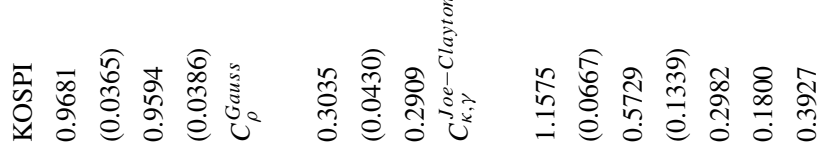

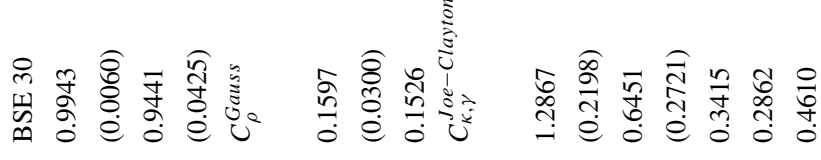

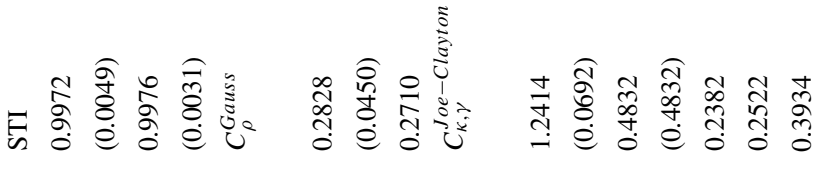

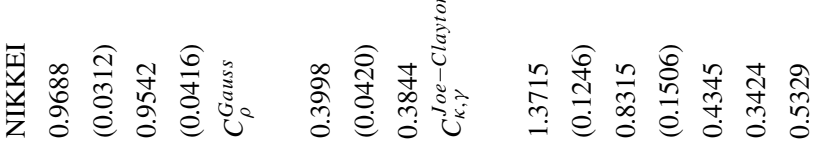

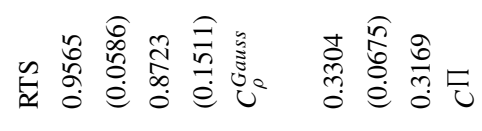

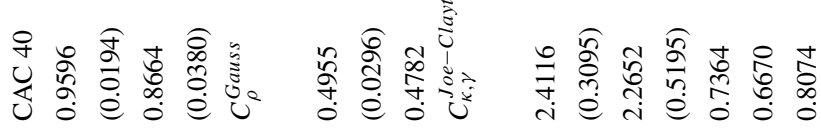
एँ

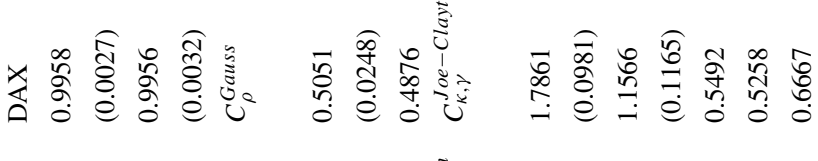

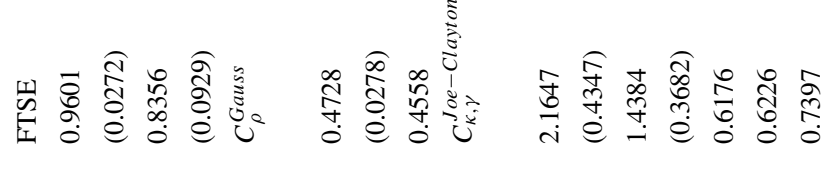

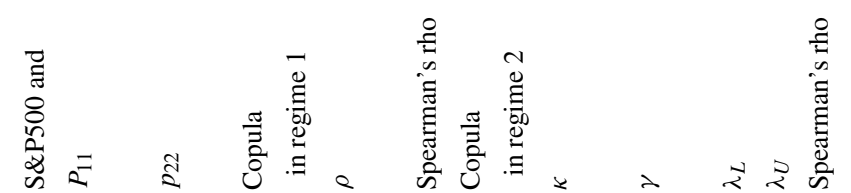


the dependence in regimes 1 and 2, with the weights equal to the predicted probabilities $P\left(S_{t}=1 \mid \Omega_{t-1}\right)$ and $P\left(S_{t}=2 \mid \Omega_{t-1}\right)$. Formulas (12) and (13) imply that an analogous property holds for the lower and upper tail dependence coefficients.

Our comparison of the mean values of the mentioned dependence measures taken in the subperiods of equal length uses the model confidence set (MCS) methodology. The ranking of the periods according to the mean value of Spearman's rho is obtained with the MCS procedure applied sequentially_after each run, the series constituting the MCS are excluded. The higher score means the stronger dependence. In the case where the MCS consists of at least two periods, the arithmetic mean of the corresponding scores is assigned to each of them. The same procedure is conducted for the series of the predicted values of tail dependence coefficients. All MCSs (with the significance level $\alpha=0.1$ ) have been estimated using the package MulCom of Hansen and Lunde (2010) written in Ox (Doornik 2006).

Maximizing jointly the log-likelihood function (24) can be very computationally intensive. Therefore, as in many papers dealing with dynamic copula models, we separate the estimation of models for marginal distributions from the estimation of the model for the dependence structure. This two-stage estimation, proposed by Joe and $\mathrm{Xu}$ (1996), is not fully efficient but there are some simulation results that motivate this approach (Patton 2009). For marginal distributions, we use ARMA-GARCH models. Since some of the analyzed periods are quite long and encompass periods of

Table 4 Linkages with the DAX: parameter estimates for the MSC models for the period 1995-2002

\begin{tabular}{|c|c|c|c|c|c|c|}
\hline DAX and & FTSE & WIG20 & RTS & NIKKEI & BSE 30 & KOSPI \\
\hline \multirow[t]{2}{*}{$P_{11}$} & 0.9896 & 0.9953 & 0.9851 & 0.9810 & 0.9949 & 0.9994 \\
\hline & $(0.0056)$ & $(0.0042)$ & $(0.0089)$ & $(0.0159)$ & $(0.0038)$ & $(0.0008)$ \\
\hline \multirow[t]{2}{*}{$P_{22}$} & 0.9828 & 0.9979 & 0.9832 & 0.9613 & 0.9931 & 0.9992 \\
\hline & $(0.0120)$ & $(0.0019)$ & $(0.0108)$ & $(0.0331)$ & $(0.0053)$ & $(0.0011)$ \\
\hline $\begin{array}{l}\text { Copula } \\
\text { in regime } 1\end{array}$ & $C_{\rho}^{\text {Gauss }}$ & $C_{\rho}^{\text {Gauss }}$ & $C_{\rho}^{\text {Gauss }}$ & $C_{\rho}^{\text {Gauss }}$ & $C^{\prod}$ & $C_{\rho}^{\text {Gauss }}$ \\
\hline \multirow[t]{2}{*}{$\rho$} & 0.5402 & 0.1180 & 0.1255 & 0.1769 & - & 0.2214 \\
\hline & $(0.0250)$ & $(0.0470)$ & $(0.0426)$ & $(0.0379)$ & & $(0.0275)$ \\
\hline Spearman's rho & 0.5223 & 0.1127 & 0.1199 & 0.1691 & 0 & 0.2118 \\
\hline $\begin{array}{l}\text { Copula } \\
\text { in regime } 2\end{array}$ & $C_{\kappa, \gamma}^{\text {Joe-Clayton }}$ & $C_{\kappa, \gamma}^{J o e-C l a y t o n}$ & $C_{\kappa, \gamma}^{\text {Joe-Clayton }}$ & $C_{\kappa, \gamma}^{J o e-C l a y t o n}$ & $C_{\kappa, \gamma}^{\text {Clayton }}$ & $C П$ \\
\hline \multirow[t]{2}{*}{$\kappa$} & 2.0218 & 1.1614 & 1.1800 & 1.2498 & 1.1509 & - \\
\hline & $(0.1851)$ & $(0.0422)$ & $(0.0765)$ & $(0.1370)$ & $(0.0537)$ & \\
\hline \multirow[t]{2}{*}{$\gamma$} & 1.3401 & 0.5585 & 0.5882 & 0.5231 & 0.2422 & - \\
\hline & $(0.1816)$ & $(0.0514)$ & (0.0999) & $(0.1346)$ & $(0.0620)$ & \\
\hline$\lambda_{L}$ & 0.5962 & 0.2890 & 0.3078 & 0.2658 & 0.0572 & 0 \\
\hline$\lambda_{U}$ & 0.5911 & 0.1836 & 0.2007 & 0.2588 & 0.1738 & 0 \\
\hline Spearman's rho & 0.7164 & 0.3890 & 0.4065 & 0.4102 & 0.2517 & 0 \\
\hline
\end{tabular}


low and high volatility, the fitted models include asymmetric GARCH (APARCH or GJR-GARCH) with a skewed Student $t$ distribution for the innovations $\varepsilon_{i, t}$. The fitted models are sometimes fractionally integrated models but this is rather a result of possible structural breaks in the data than long memory effects (Beine and Laurent 2001).

The standardized residuals $\hat{\varepsilon}_{i, t}$ from the fitted models were routinely examined for the presence of desirable distributional and serial properties. Next, they were transformed by means of the corresponding distribution functions into the series of the form (23). The parameters of the MSC models were estimated by the maximum likelihood method, using the first summand in (24). In order to model adequately the dynamics of the linkages, we tried to use copulas from various families. As it was mentioned above, the best models were selected using information criteria, and likelihood ratio tests in the cases where nested models were compared.

In Tables 2 and 3 we present the estimation results for the linkages with the S\&P 500 for the case where the total sample period is partitioned into two subperiods: 1995-2002 and 2002-2009. The tables include parameter estimates of the fitted MSC models, the values of Spearman's rho for copulas prevailing in a given regime, and the values of the coefficients of tail dependence (where applicable). Tables 4 and 5 show the corresponding results for the linkages between the DAX and the indices FTSE, WIG20, RTS, NIKKEI, BSE 30, and KOSPI. Due to space limitations, we

Table 5 Linkages with the DAX: parameter estimates for the MSC models for the period 2002-2009

\begin{tabular}{|c|c|c|c|c|c|c|}
\hline DAX and & FTSE & WIG20 & RTS & NIKKEI & BSE 30 & KOSPI \\
\hline \multirow[t]{2}{*}{$p_{11}$} & 0.9808 & 0.9994 & 0.9852 & 0.9436 & 0.9992 & 0.9748 \\
\hline & $(0.0080)$ & $(0.0008)$ & $(0.0086)$ & $(0.0698)$ & $(0.0011)$ & $(0.0288)$ \\
\hline \multirow[t]{2}{*}{$p_{22}$} & 0.9689 & 0.9994 & 0.9809 & 0.8887 & 0.9990 & 0.8936 \\
\hline & $(0.0110)$ & $(0.0007)$ & $(0.0106)$ & $(0.1116)$ & $(0.0016)$ & $(0.1089)$ \\
\hline $\begin{array}{l}\text { Copula } \\
\text { in regime } 1\end{array}$ & $C_{\rho}^{\text {Gauss }}$ & $C_{\rho}^{\text {Gauss }}$ & $C_{\rho}^{\text {Gauss }}$ & $C_{\rho}^{\text {Gauss }}$ & $C_{\rho}^{\text {Gauss }}$ & $C_{\rho}^{\text {Gauss }}$ \\
\hline \multirow[t]{2}{*}{$\rho$} & 0.7859 & 0.6432 & 0.2113 & 0.2438 & 0.4103 & 0.2887 \\
\hline & $(0.0135)$ & $(0.0181)$ & $(0.0483)$ & $(0.0445)$ & $(0.0268)$ & $(0.0320)$ \\
\hline Spearman's rho & 0.7713 & 0.6253 & 0.2021 & 0.2334 & 0.3946 & 0.2767 \\
\hline $\begin{array}{l}\text { Copula } \\
\text { in regime } 2\end{array}$ & $C_{\rho}^{\text {Gauss }}$ & $C_{\gamma}^{\text {Clayton }}$ & $C_{\rho}^{\text {Gauss }}$ & $C_{\kappa, \gamma}^{J o e-C l a y t o n}$ & $C_{\gamma}^{\text {Clayton }}$ & $C_{\gamma}^{\text {Clayton }}$ \\
\hline \multirow[t]{2}{*}{$\kappa$ or $\rho$} & 0.9284 & - & 0.6221 & 1.2262 & - & - \\
\hline & $(0.0084)$ & & $(0.0358)$ & $(0.1159)$ & & \\
\hline \multirow[t]{2}{*}{$\gamma$} & - & 0.6055 & - & 0.5755 & 0.2530 & 0.8913 \\
\hline & & $(0.0531)$ & $(0.1972)$ & $(0.0700)$ & $(0.3149)$ & \\
\hline$\lambda_{L}$ & - & 0.3183 & - & 0.2999 & 0.0646 & 0.4595 \\
\hline$\lambda_{U}$ & - & - & - & 0.2400 & - & - \\
\hline Spearman's rho & 0.9219 & 0.3408 & 0.6041 & 0.4191 & 0.1675 & 0.4451 \\
\hline
\end{tabular}




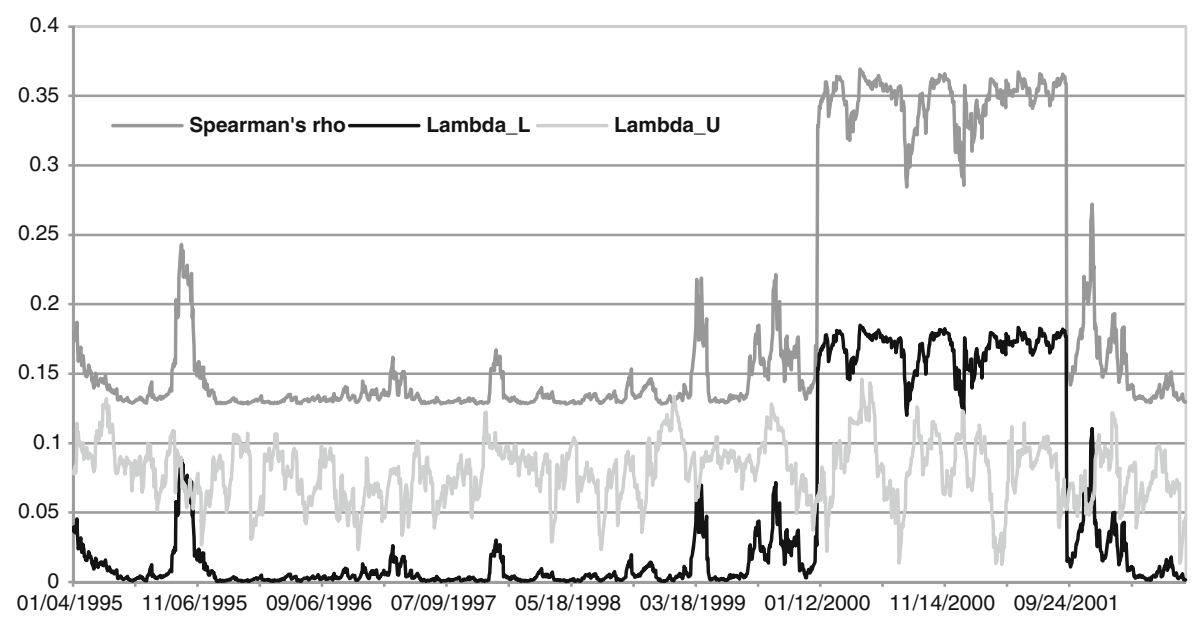

Fig. 1 S\&P 500 and KOSPI. Period 1995-2002. Predicted values for Spearman's rho and tail dependence coefficients

do not report parameter estimates of the remaining 432 MSC models corresponding to the finer partitions of the total time period. Based on these models we obtained rankings of the linkages presented in Tables 7, 8, 9, 11, 12 and 13.

The MSC models presented in Tables 2 and 3 fit well the data. The only exceptions in which a non-switching model is selected are the WIG20 (in both subperiods) and the STI. In the case of the RTS, in both subperiods one of the regimes is govern by the independence copula. The values of Spearman's rho are always higher in regimes where tail dependence is present.

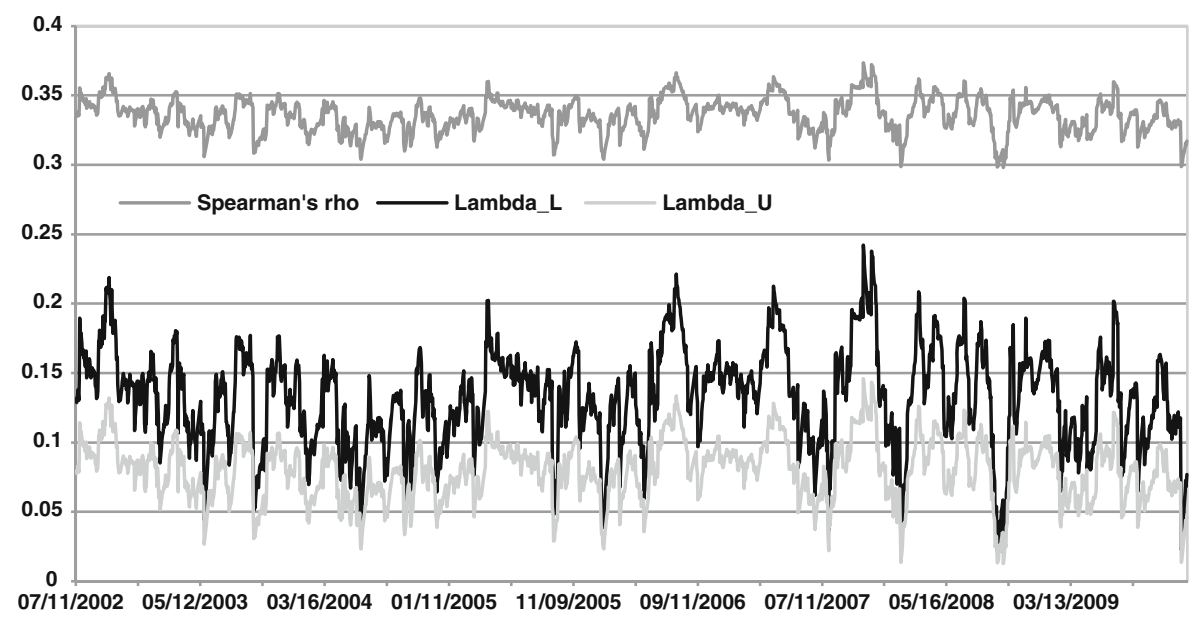

Fig. 2 S\&P 500 and KOSPI. Period 2002-2009. Predicted values for Spearman's rho and tail dependence coefficients 


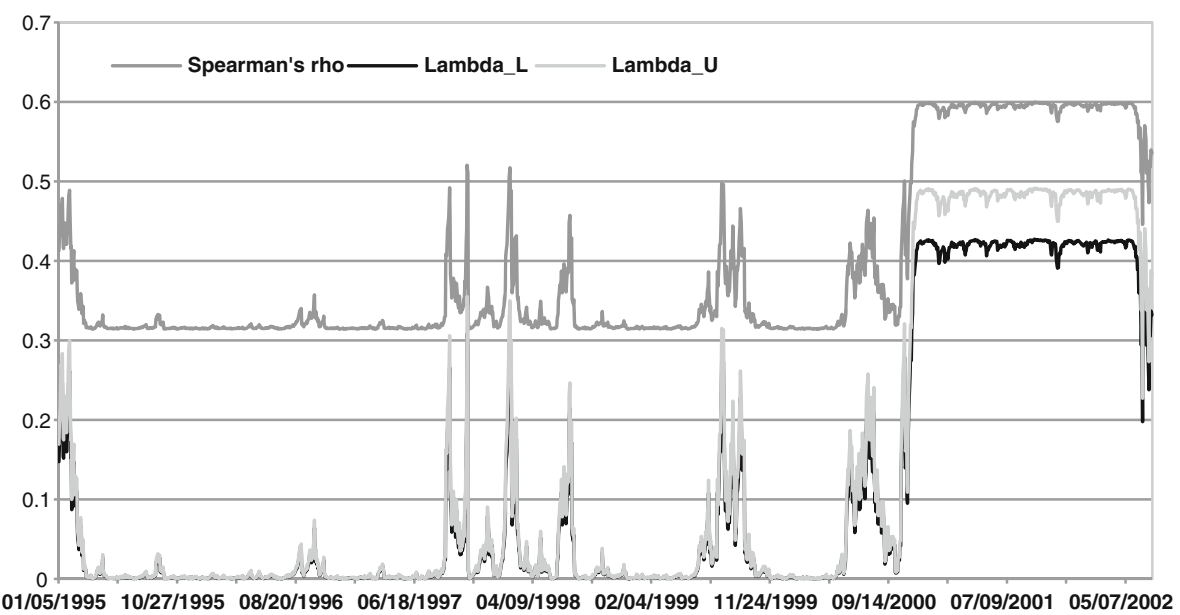

Fig. 3 DAX and S\&P 500. Period 1995-2002. Predicted values for Spearman's rho and tail dependence coefficients

The results for the DAX (Tables 3 and 4) are generally similar to those for the S\&P 500. There are two cases where Spearman's rhos calculated for a copula with tail dependence are lower than for the Gaussian copula. This is true for the pairs (DAX,WIG20) and (DAX, BSE 30) in the second subperiod. Usually, it is expected that during a crisis period the dependencies are stronger and that this is accompanied by an increase in tail dependence. The above examples show that the strength of regular linkages in a regime with no tail dependence can be higher than in a regime where tail dependence is present.

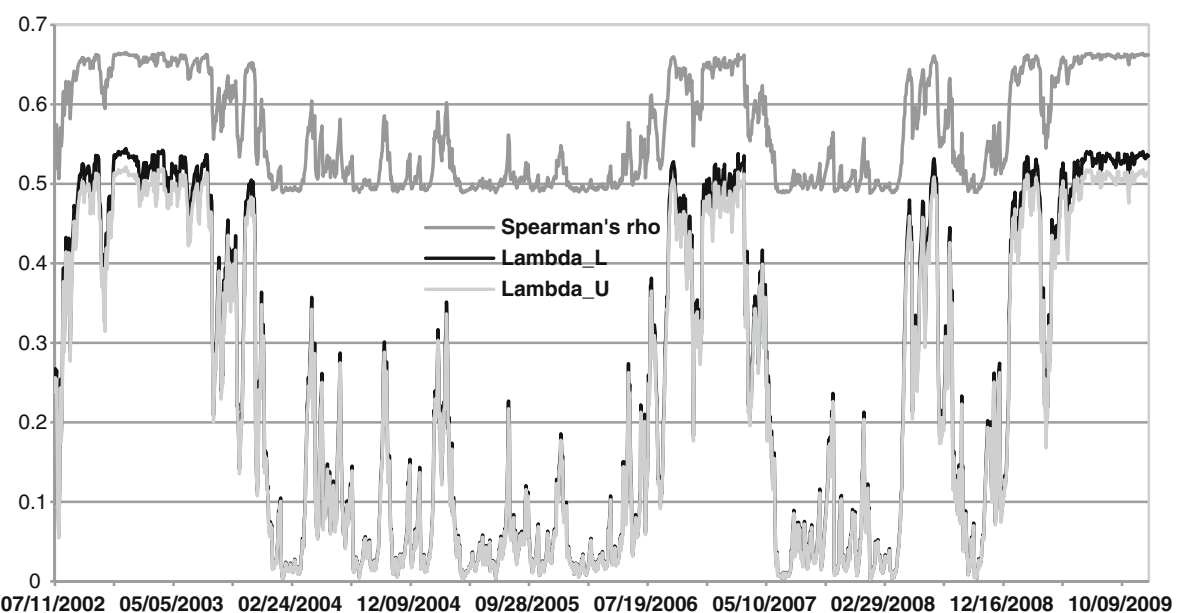

Fig. 4 DAX and S\&P 500. Period 2002-2009. Predicted values for Spearman's rho and tail dependence coefficients 
Table 6 The ranking of the strength of linkages with S\&P 500: two subperiods

\begin{tabular}{|c|c|c|c|c|c|c|c|c|c|c|}
\hline S\&P 500 and & FTSE & DAX & $\mathrm{CAC} 40$ & WIG20 & RTS & NIKKEI & STI & BSE30 & KOSPI & BOVESPA \\
\hline \multicolumn{11}{|c|}{ Spearman's rho } \\
\hline 1995-2002 & 1 & 1 & 1 & 2 & 1 & 1 & 1 & 1 & 1 & 1 \\
\hline 2002-2009 & 2 & 2 & 2 & 1 & 2 & 2 & 2 & 2 & 2 & 2 \\
\hline \multicolumn{11}{|l|}{ Lambda L } \\
\hline 1995-2002 & 2 & 1 & 1 & 2 & 2 & 1 & 1 & 1 & 1 & 1 \\
\hline 2002-2009 & 1 & 2 & 2 & 1 & 1 & 2 & 2 & 2 & 2 & 2 \\
\hline \multicolumn{11}{|l|}{ Lambda U } \\
\hline 1995-2002 & 2 & 1 & 1.5 & 2 & 2 & 1 & 1 & 1 & 1 & 1 \\
\hline 2002-2009 & 1 & 2 & 1.5 & 1 & 1 & 2 & 2 & 2 & 2 & 2 \\
\hline
\end{tabular}

To give a reader an impression of the dependence dynamics we present in Figs. 1 and 2 the plots of the predicted values of Spearman's rho and the tail dependence coefficients for the S\&P 500 and KOSPI in periods 1995-2002 and 2002-2009, respectively. Figures 3 and 4 show the corresponding plots for the pair (DAX, S\&P 500).

Tables $6,7,8,9,10,11,12$ and 13 present the rankings of subperiods obtained as a result of comparison of strength of linkages between the considered indices and the S\&P 500 or the DAX. The idea behind our analysis is that increase in the strength of linkages during longer subperiods (Tables 6, 7, 10 and 11) can be interpreted as the effect of globalization. The analysis performed for shorter

Table 7 The ranking of the strength of linkages with S\&P 500: four subperiods

\begin{tabular}{|c|c|c|c|c|c|c|c|c|c|c|}
\hline S\&P 500 and & FTSE & DAX & CAC40 & WIG20 & RTS & NIKKEI & STI & BSE30 & KOSPI & BOVESPA \\
\hline \multicolumn{11}{|c|}{ Spearman's rho } \\
\hline 1995-1998 & 1 & 1 & 1 & 1 & 3 & 1 & 1 & 1 & 1 & 1 \\
\hline 1998-2002 & 2 & 2 & 2 & 4 & 2 & 2 & 3 & 2 & 2 & 3 \\
\hline 2002-2006 & 3 & 3 & 3 & 3 & 1 & 3 & 2 & 3 & 3 & 2 \\
\hline 2006-2009 & 4 & 4 & 4 & 2 & 4 & 4 & 4 & 4 & 4 & 4 \\
\hline \multicolumn{11}{|l|}{ Lambda L } \\
\hline 1995-1998 & 1 & 1 & 1 & 3 & 1 & 1 & 1 & 2 & 1 & 3 \\
\hline 1998-2002 & 4 & 2 & 3 & 1 & 4 & 3 & 4 & 3 & 3 & 1.5 \\
\hline 2002-2006 & 3 & 3 & 4 & 4 & 3 & 2 & 2 & 4 & 2 & 4 \\
\hline 2006-2009 & 2 & 4 & 2 & 2 & 2 & 4 & 3 & 1 & 4 & 1.5 \\
\hline \multicolumn{11}{|l|}{ Lambda U } \\
\hline 1995-1998 & 1 & 1 & 1 & 4 & 2.5 & 2 & 1 & 1.5 & 1 & 3 \\
\hline 1998-2002 & 4 & 2 & 4 & 2 & 2.5 & 2 & 2 & 1.5 & 4 & 1.5 \\
\hline 2002-2006 & 3 & 3.5 & 3 & 2 & 2.5 & 2 & 3 & 1.5 & 2 & 4 \\
\hline 2006-2009 & 2 & 3.5 & 2 & 2 & 2.5 & 4 & 4 & 1.5 & 3 & 1.5 \\
\hline
\end{tabular}


Table 8 The ranking of the strength of linkages with S\&P500: eight periods

S\&P 500 and FTSE DAX CAC40 WIG20 RTS NIKKEI STI BSE30 KOSPI BOVESPA

\begin{tabular}{clllllllllll}
\hline $\begin{array}{c}\text { Spearman's rho } \\
1995-1996\end{array}$ & 1 & 1 & 1 & 1 & 3.5 & 2 & 2 & 1 & 1 & 1 \\
$1997-1998$ & 3 & 3 & 3 & 6 & 7 & 1 & 1 & 3 & 2 & 2 \\
$1998-2000$ & 2 & 2 & 2 & 3 & 1.5 & 4 & 5 & 2 & 4 & 5.5 \\
$2000-2002$ & 6 & 7 & 5.5 & 7 & 3.5 & 3 & 6 & 6 & 5 & 4 \\
$2002-2004$ & 5 & 6 & 5.5 & 4 & 1.5 & 5 & 3 & 5 & 7 & 3 \\
$2004-2006$ & 4 & 4 & 4 & 8 & 5 & 6.5 & 4 & 4 & 3 & 5.5 \\
$2006-2007$ & 7 & 5 & 7 & 5 & 8 & 6.5 & 8 & 7 & 8 & 8 \\
$2008-2009$ & 8 & 8 & 8 & 2 & 6 & 8 & 7 & 8 & 6 & 7
\end{tabular}

Lambda L

$\begin{array}{lllllllllll}1995-1996 & 4 & 1 & 2 & 2 & 6 & 7 & 3.5 & 3 & 2.5 & 5.5 \\ 1997-1998 & 5 & 3.5 & 4 & 5 & 8 & 3 & 3.5 & 5 & 2.5 & 5.5 \\ 1998-2000 & 6 & 3.5 & 5 & 4 & 5 & 3 & 8 & 4 & 6 & 2.5 \\ 2000-2002 & 8 & 6 & 2 & 1 & 3 & 3 & 3.5 & 6 & 2.5 & 2.5 \\ 2002-2004 & 3 & 8 & 8 & 7 & 1 & 3 & 7 & 8 & 2.5 & 7 \\ 2004-2006 & 7 & 2 & 2 & 8 & 4 & 3 & 3.5 & 7 & 5 & 2.5 \\ 2006-2007 & 1.5 & 5 & 6 & 6 & 2 & 6 & 3.5 & 1 & 7.5 & 2.5 \\ 2008-2009 & 1.5 & 7 & 7 & 3 & 7 & 8 & 3.5 & 2 & 7.5 & 8\end{array}$

Lambda U

$\begin{array}{llllllllllll}1995-1996 & 2 & 1.5 & 2.5 & 7 & 3.5 & 4 & 3 & 7 & 6 & 3 \\ 1997-1998 & 4 & 1.5 & 5 & 6 & 8 & 4 & 6 & 3 & 2.5 & 7 \\ 1998-2000 & 5 & 3 & 6 & 5 & 3.5 & 4 & 3 & 6 & 5 & 3 \\ 2000-2002 & 8 & 6 & 2.5 & 2.5 & 3.5 & 4 & 3 & 8 & 2.5 & 3 \\ 2002-2004 & 6 & 8 & 8 & 2.5 & 3.5 & 4 & 3 & 3 & 2.5 & 6 \\ 2004-2006 & 7 & 4 & 2.5 & 8 & 3.5 & 4 & 8 & 3 & 7 & 3 \\ 2006-2007 & 2 & 5 & 2.5 & 2.5 & 3.5 & 8 & 3 & 3 & 8 & 3 \\ 2008-2009 & 2 & 7 & 7 & 2.5 & 7 & 4 & 7 & 3 & 2.5 & 8\end{array}$

Shaded fields indicate crises periods

periods (Tables 8, 9, 12 and 13) allows us to discover the impact of market events (especially crises) on the strength of dependence. The rankings for Spearman's rho show the behavior of regular dependence and the rankings calculated based on the tail dependence coefficients describe the changes of dependence in extremes.

The first observations is that the rankings depend on the applied measure of dependence. The results obtained for Spearman's rho are in favor of the globalization effect. Tables 6 and 10 show that in the case of partitioning into two subperiods an increase in the strength of dependencies between the markets is visible. The only and easy to explain exception is the pair (S\&P 500, WIG20). 
Table 9 The ranking of the linkages with S\&P500: fifteen periods (years)

S\&P 500 and FTSE DAX CAC 40 WIG20 RTS NIKKEI STI BSE 30 KOSPI BOVESPA

\begin{tabular}{lcccccccccc}
\hline Spearman's rho & & & & & & & & & & \\
1995 & 1.5 & 1 & 1 & 1 & - & 1.5 & 6.5 & 6 & 2.5 & 2 \\
1996 & 1.5 & 2 & 2.5 & 5 & 1 & 3.5 & 1 & 1 & 1 & 1 \\
1997 & 3 & 3 & 4 & 9 & 14 & 3.5 & 2.5 & 5 & 2.5 & 3 \\
1998 & 8 & 6 & 5 & 12 & 3 & 1.5 & 2.5 & 4 & 4 & 8 \\
1999 & 4 & 6 & 7.5 & 3 & 7.5 & 5.5 & 9.5 & 3 & 5 & 5.5 \\
2000 & 5 & 6 & 2.5 & 15 & 7.5 & 12 & 8 & 8 & 15 & 11 \\
2001 & 12 & 14 & 11.5 & 10 & 5 & 7 & 11 & 12 & 12 & 7 \\
2002 & 12 & 12.5 & 11.5 & 7 & 7.5 & 8 & 5 & 2 & 7.5 & 4 \\
2003 & 10 & 12.5 & 9 & 6 & 2 & 9 & 4 & 10 & 12 & 9 \\
2004 & 7 & 6 & 6 & 11 & 4 & 10.5 & 6.5 & 7 & 6 & 10 \\
2005 & 6 & 6 & 7.5 & 14 & 11 & 10.5 & 12 & 9 & 7.5 & 5.5 \\
2006 & 12 & 9.5 & 13.5 & 8 & 12 & 5.5 & 14.5 & 11 & 9.5 & 13 \\
2007 & 14 & 9.5 & 13.5 & 4 & 13 & 13 & 13 & 13.5 & 14 & 14 \\
2008 & 9 & 11 & 10 & 13 & 10 & 14 & 14.5 & 13.5 & 12 & 12 \\
2009 & 15 & 15 & 15 & 2 & 7.5 & 15 & 9.5 & 15 & 9.5 & 15
\end{tabular}

Lambda L

$\begin{array}{lcccccccccc}1995 & 14 & 4 & 4.5 & 3.5 & - & 12 & 3.5 & 5 & 4.5 & 4.5 \\ 1996 & 4.5 & 2 & 4.5 & 9 & 7 & 14 & 8 & 6 & 4.5 & 11.5 \\ 1997 & 4.5 & 9 & 9.5 & 13 & 13 & 5.5 & 9.5 & 10 & 4.5 & 8.5 \\ 1998 & 4.5 & 5 & 4.5 & 3.5 & 8 & 5.5 & 11.5 & 9 & 4.5 & 4.5 \\ 1999 & 4.5 & 2 & 4.5 & 8 & 13 & 12 & 15 & 8 & 4.5 & 4.5 \\ 2000 & 11 & 10.5 & 9.5 & 3.5 & 3.5 & 5.5 & 9.5 & 13 & 9.5 & 4.5 \\ 2001 & 15 & 6 & 12 & 3.5 & 3.5 & 5.5 & 3.5 & 11 & 13 & 8.5 \\ 2002 & 11 & 10.5 & 13 & 11 & 11 & 5.5 & 3.5 & 7 & 9.5 & 4.5 \\ 2003 & 11 & 15 & 15 & 10 & 3.5 & 5.5 & 3.5 & 15 & 4.5 & 11.5 \\ 2004 & 4.5 & 7.5 & 11 & 15 & 9 & 5.5 & 7 & 12 & 4.5 & 13 \\ 2005 & 11 & 2 & 4.5 & 14 & 13 & 5.5 & 13 & 14 & 14 & 14.5 \\ 2006 & 4.5 & 13 & 4.5 & 3.5 & 10 & 5.5 & 11.5 & 3 & 15 & 10 \\ 2007 & 4.5 & 12 & 4.5 & 12 & 3.5 & 15 & 14 & 3 & 11.5 & 4.5 \\ 2008 & 4.5 & 7.5 & 4.5 & 3.5 & 3.5 & 12 & 3.5 & 3 & 11.5 & 14.5 \\ 2009 & 13 & 14 & 14 & 7 & 3.5 & 5.5 & 3.5 & 3 & 4.5 & 4.5\end{array}$

Lambda U

$\begin{array}{lcccrrrrrrr}1995 & 5.5 & 3.5 & 5.5 & 13 & - & 12 & 5.5 & 14 & 4.5 & 5.5 \\ 1996 & 5.5 & 3.5 & 5.5 & 6 & 7 & 13 & 5.5 & 6.5 & 9 & 5.5 \\ 1997 & 5.5 & 8 & 11 & 14 & 14 & 6 & 5.5 & 6.5 & 4.5 & 11 \\ 1998 & 5.5 & 7 & 5.5 & 6 & 7 & 6 & 11 & 13 & 4.5 & 5.5 \\ 1999 & 5.5 & 3.5 & 5.5 & 6 & 7 & 14 & 5.5 & 6.5 & 4.5 & 5.5 \\ 2000 & 12 & 10 & 5.5 & 6 & 7 & 6 & 12 & 6.5 & 14 & 5.5\end{array}$


Table 9 (continued)

\begin{tabular}{lcccccccccc}
\hline S\&P 500 and & FTSE & DAX & CAC 40 & WIG20 & RTS & NIKKEI & STI & BSE 30 & KOSPI & BOVESPA \\
\hline 2001 & 15 & 3.5 & 5.5 & 6 & 7 & 6 & 5.5 & 15 & 13 & 5.5 \\
2002 & 13.5 & 15 & 13.5 & 6 & 7 & 6 & 5.5 & 6.5 & 10 & 5.5 \\
2003 & 5.5 & 13 & 13.5 & 6 & 7 & 6 & 14 & 6.5 & 4.5 & 5.5 \\
2004 & 5.5 & 3.5 & 15 & 6 & 7 & 6 & 5.5 & 6.5 & 4.5 & 12 \\
2005 & 13.5 & 3.5 & 5.5 & 15 & 7 & 6 & 13 & 6.5 & 4.5 & 14 \\
2006 & 5.5 & 14 & 5.5 & 6 & 7 & 6 & 15 & 6.5 & 12 & 13 \\
2007 & 5.5 & 10 & 5.5 & 6 & 7 & 15 & 5.5 & 6.5 & 15 & 5.5 \\
2008 & 5.5 & 10 & 5.5 & 6 & 7 & 6 & 5.5 & 6.5 & 11 & 15 \\
2009 & 11 & 12 & 12 & 12 & 7 & 6 & 5.5 & 6.5 & 4.5 & 5.5 \\
\hline
\end{tabular}

Shaded fields indicate crises periods

The Polish stock market is traditionally strongly connected with the U.S. one. The EU joining process, however, resulted in weakening these linkages due to a natural effect of approaching the Polish economy the EU one. In the case of four subperiods (Tables 7 and 11), more exceptions is observed, but generally the rankings indicate a more or less regular increase in the strength of connections.

The results concerning dependencies in tails are more diverse. Although very often higher score corresponds to the period 2002-2009, there are some interesting exceptions. The first of them is the pair (S\&P 500, FTSE) for which our results indicate weakening of tail dependencies in the second period. Similar results were obtained for the pairs (S\&P 500, RTS), (DAX, FTSE), (DAX, WIG20), (DAX, RTS), and, with reference to upper tail dependence, for (DAX, NIKKEI) and (DAX, BSE 30). These results show that the globalization effect does not necessarily mean an increase in the strength of tail dependencies, and again that the behavior of regular dependence measured by means of Spearman's rho can be different from that of dependence in

Table 10 The ranking of the strength of linkages with DAX: two subperiods

\begin{tabular}{|c|c|c|c|c|c|c|}
\hline DAX and & FTSE & WIG20 & RTS & NIKKEI & BSE 30 & KOSPI \\
\hline \multicolumn{7}{|c|}{ Spearman's rho } \\
\hline 1995-2002 & 1 & 1 & 1 & 1 & 1 & 1 \\
\hline 2002-2009 & 2 & 2 & 2 & 2 & 2 & 2 \\
\hline \multicolumn{7}{|l|}{ Lambda L } \\
\hline 1995-2002 & 2 & 2 & 2 & 1 & 1 & 1 \\
\hline 2002-2009 & 1 & 1 & 1 & 2 & 2 & 2 \\
\hline \multicolumn{7}{|l|}{ Lambda U } \\
\hline 1995-2002 & 2 & 2 & 2 & 2 & 2 & 1 \\
\hline 2002-2009 & 1 & 1 & 1 & 1 & 1 & 2 \\
\hline
\end{tabular}


Table 11 The ranking of the strength of linkages with DAX: four subperiods

\begin{tabular}{lllllll}
\hline DAX and & FTSE & WIG20 & RTS & NIKKEI & BSE 30 & KOSPI \\
\hline Spearman's rho & & & & & & \\
$1995-1998$ & 1 & 1 & 1 & 2 & 1 & 1 \\
$1998-2002$ & 2 & 3 & 3 & 1 & 2 & 2 \\
$2002-2006$ & 3 & 2 & 2 & 3 & 3 & 3 \\
$2006-2009$ & 4 & 4 & 4 & 4 & 4 & 4 \\
Lambda L & & & & & & \\
$1995-1998$ & 3.5 & 1 & 4 & 2 & 3 & 1 \\
$1998-2002$ & 3.5 & 3 & 3 & 4 & 1 & 2 \\
$2002-2006$ & 1 & 4 & 2 & 3 & 4 & 4 \\
$2006-2009$ & 2 & 2 & 1 & 1 & 2 & 3 \\
Lambda U & & & & & & \\
$1995-1998$ & 3 & 2 & 3 & 2 & 3 & 2.5 \\
$1998-2002$ & 4 & 4 & 4 & 2 & 1 & 2.5 \\
$2002-2006$ & 1 & 2 & 1 & 4 & 4 & 2.5 \\
$2006-2009$ & 2 & 2 & 2 & 2 & 2 & 2.5 \\
\hline
\end{tabular}

extremes. Moreover, the rankings obtained based on the dynamics of the lower and upper tail dependence coefficients often differ from each other.

The period under scrutiny includes two subperiods in which severe financial crises appeared. In years 1997-1998 the financial market experienced a turmoil starting from emerging economies, and from 2007 to 2009 it suffered from the U.S. subprime crisis. The analysis of Tables 8, 9,12 and 13 allows one to explore the issue of increasing strength of market dependencies during the crisis periods. Although none of the analyzed subperiods can be perceived as a crisis period, a systemic increase in the strength of dependence during crises should result in a significantly higher level of dependence for periods encompassing a crisis. In the analysis we have to take into account effects of globalization, thus, for example, we do not expect the level of dependence in the period 1997-1998 to be the highest, but if there were an increase in the strength of linkages during the crisis, the level should be the highest from among those in the first four periods considered in Table 8 or in the first seven periods in Table 12. Hence our results do not, as a rule, indicate an increase in the strength of dependence during the crisis periods. The results are rather ambiguous and depend on the analyzed markets. For example, Table 8 shows that in years 1997-1998 the linkages between the S\&P and the Polish WIG20 belong to the highest, but in years 2007-2009 are located among the lowest. In the case of the S\&P and RTS, the scores for these two subperiods are very close. This diversity of results is observed in the case of all the considered measures of dependence.

An interesting observation supporting the use of the MCS methodology is that we find pairs of the indices with the same score for different periods with a non-zero level of dependence. The examples are: (S\&P 500, CAC 40) (Tables 6, 8 and 9), (S\&P 
Table 12 The ranking of the strength of linkages with DAX: eight subperiods

\begin{tabular}{|c|c|c|c|c|c|c|}
\hline DAX and & FTSE & WIG20 & RTS & NIKKEI & BSE 30 & KOSPI \\
\hline \multicolumn{7}{|c|}{ Spearman's rho } \\
\hline 1995-1996 & 1 & 1 & 1 & 6 & 2 & 1 \\
\hline 1997-1998 & 2 & 2.5 & 3 & 1 & 3.5 & 2 \\
\hline 1998-2000 & 3 & 6 & 6 & 2.5 & 1 & 3 \\
\hline $2000-2002$ & 4 & 4 & 5 & 2.5 & 5 & 4 \\
\hline 2002-2004 & 5 & 5 & 2 & 4 & 3.5 & 5 \\
\hline 2004-2006 & 6 & 2.5 & 4 & 7.5 & 6 & 6.5 \\
\hline 2006-2007 & 7 & 7 & 8 & 7.5 & 7 & 6.5 \\
\hline 2008-2009 & 8 & 8 & 7 & 5 & 8 & 8 \\
\hline \multicolumn{7}{|l|}{ Lambda L } \\
\hline 1995-1996 & 8 & 2 & 3.5 & 2 & 3 & 5 \\
\hline 1997-1998 & 7 & 4 & 7 & 4 & 7 & 2.5 \\
\hline 1998-2000 & 6 & 6 & 3.5 & 7 & 6 & 2.5 \\
\hline $2000-2002$ & 3 & 2 & 3.5 & 2 & 3 & 2.5 \\
\hline $2002-2004$ & 3 & 7 & 3.5 & 5.5 & 8 & 6 \\
\hline 2004-2006 & 3 & 8 & 3.5 & 8 & 3 & 7 \\
\hline 2006-2007 & 3 & 5 & 3.5 & 5.5 & 3 & 2.5 \\
\hline 2008-2009 & 3 & 2 & 8 & 2 & 3 & 8 \\
\hline \multicolumn{7}{|l|}{ Lambda U } \\
\hline 1995-1996 & 4 & 4 & 4 & 4 & 4 & 6 \\
\hline 1997-1998 & 8 & 4 & 4 & 4 & 4 & 3 \\
\hline 1998-2000 & 4 & 4 & 4 & 4 & 8 & 3 \\
\hline $2000-2002$ & 4 & 4 & 4 & 4 & 4 & 3 \\
\hline 2002-2004 & 4 & 8 & 4 & 8 & 4 & 7.5 \\
\hline 2004-2006 & 4 & 4 & 4 & 4 & 4 & 3 \\
\hline 2006-2007 & 4 & 4 & 4 & 4 & 4 & 3 \\
\hline 2008-2009 & 4 & 4 & 8 & 4 & 4 & 7.5 \\
\hline
\end{tabular}

Shaded fields indicate crises periods

500, BOVESPA) (Table 8) and (DAX, NIKKEI) (Tables 12 and 13). Thus different dynamics can result in statistically indistinguishable average levels of dependence.

Our findings can be summarized as follows. First, an observed increase in the strength of market linkages is due rather to the globalization, and not specifically to crises. In fact, in the considered crisis periods no general increase in dependence measured by Spearman's rho is detected. Moreover, what is even more surprising, we do not observe any systemic higher level of lower tail dependence during the crises. Another unexpected result is that for some pairs of the indices upper tail dependence turns out to be greater than the lower one. An example for this is shown in Fig. 1. 
Table 13 The ranking of the strength of linkages with DAX: fifteen periods (years)

\begin{tabular}{lcccccc}
\hline DAX and & FTSE & WIG20 & RTS & NIKKEI & BSE 30 & KOSPI \\
\hline Spearman's rho & & & & & & \\
1995 & 2 & 1 & - & 4 & 2.5 & 3 \\
1996 & 1 & 2 & 1 & 14 & 1 & 2 \\
1997 & 3 & 3.5 & 2 & 2.5 & 6 & 1 \\
1998 & 6 & 11.5 & 9.5 & 6.5 & 5 & 4 \\
1999 & 5 & 10 & 7.5 & 2.5 & 2.5 & 5 \\
2000 & 4 & 6 & 6 & 6.5 & 7 & 10 \\
2001 & 9 & 8.5 & 7.5 & 5 & 9 & 9 \\
2002 & 7 & 5 & 9.5 & 9 & 4 & 6 \\
2003 & 8 & 7 & 3 & 8 & 8 & 11 \\
2004 & 11 & 11.5 & 4.5 & 15 & 11 & 14.5 \\
2005 & 10 & 3.5 & 4.5 & 11 & 10 & 8 \\
2006 & 12 & 8.5 & 11 & 10 & 12 & 7 \\
2007 & 13.5 & 13 & 12 & 13 & 13 & 14.5 \\
2008 & 13.5 & 15 & 13.5 & 12 & 15 & 13 \\
2009 & 15 & 14 & 13.5 & 1 & 14 & 12
\end{tabular}

Lambda L

\begin{tabular}{ccccccc}
1995 & 4.5 & 3.5 & - & 7 & 4.5 & 10 \\
1996 & 12 & 7 & 9 & 11 & 8 & 7 \\
1997 & 4.5 & 3.5 & 4.5 & 12.5 & 4.5 & 8.5 \\
1998 & 11 & 10.5 & 14 & 3.5 & 14 & 3.5 \\
1999 & 9 & 3.5 & 4.5 & 10 & 9 & 3.5 \\
2000 & 13 & 12 & 12 & 3.5 & 4.5 & 3.5 \\
2001 & 4.5 & 3.5 & 4.5 & 3.5 & 11 & 3.5 \\
2002 & 15 & 14 & 4.5 & 3.5 & 10 & 3.5 \\
2003 & 4.5 & 15 & 4.5 & 8 & 13 & 13 \\
2004 & 14 & 3.5 & 10 & 14 & 4.5 & 14 \\
2005 & 4.5 & 13 & 4.5 & 3.5 & 12 & 15 \\
2006 & 4.5 & 8 & 4.5 & 3.5 & 15 & 11.5 \\
2007 & 4.5 & 9 & 11 & 12.5 & 4.5 & 3.5 \\
2008 & 4.5 & 10.5 & 4.5 & 15 & 4.5 & 11.5 \\
2009 & 10 & 3.5 & 13 & 9 & 4.5 & 8.5 \\
Lambda U & & & & & & \\
1995 & 6 & 6.5 & - & 13 & 5.5 & 10 \\
1996 & 6 & 6.5 & 10 & 15 & 13 & 12 \\
1997 & 6 & 6.5 & 5 & 11 & 5.5 & 9 \\
1998 & 6 & 6.5 & 11 & 5 & 5.5 & 4.5 \\
\hline 1999 & 6 & 6.5 & 5 & 5 & 11 & 4.5
\end{tabular}


Table 13 (continued)

\begin{tabular}{lcccccc}
\hline DAX and & FTSE & WIG20 & RTS & NIKKEI & BSE 30 & KOSPI \\
\hline 2000 & 14 & 6.5 & 5 & 5 & 5.5 & 4.5 \\
2001 & 6 & 6.5 & 5 & 5 & 15 & 4.5 \\
2002 & 15 & 6.5 & 5 & 5 & 12 & 4.5 \\
2003 & 6 & 13 & 5 & 14 & 5.5 & 13 \\
2004 & 13 & 6.5 & 12 & 12 & 5.5 & 14 \\
2005 & 6 & 6.5 & 5 & 5 & 5.5 & 4.5 \\
2006 & 6 & 6.5 & 5 & 5 & 5.5 & 4.5 \\
2007 & 6 & 14 & 14 & 5 & 5.5 & 4.5 \\
2008 & 6 & 15 & 5 & 10 & 5 & 11 \\
2009 & 12 & 6.5 & 13 & & 5 \\
\hline
\end{tabular}

Shaded fields indicate crises periods

\section{Conclusions}

The aim of the presented analysis was to examine the impact of globalization and crises on the strength of linkages between stock markets. Our analysis was performed for pairs of national stock markets represented by the daily returns on stock indices including the S\&P 500, DAX, FTSE, CAC 40, RTS, WIG 20, NIKKEI, STI, BSE, and BOVESPA. We considered 10 pairs containing the S\&P 500, and 6 holding the DAX. The applied approach used a Markov switching copula model to describe the dynamics of dependencies during subperiods of the period 1995-2009. We considered two types of dependencies: regular dependence measured by means of the conditional Spearman's rho, and dependencies in extremes quantified by the conditional tail dependence coefficients. To compare the strength of linkages in different subperiods the Model Confidence Set methodology was applied. Our main results are the following. In the case of long subperiods (4, 7 years), a systematic and significant increase over time in the mean level of the linkages (measured by Spearman's rho) between the national stock markets is observed. This can be interpreted as an effect of globalization. On the other hand, the globalization effect does not necessarily cause a systematic increase in the strength of tail dependencies. The mean level of dependence in shorter subperiods (1-2 years) is sensitive to the market situation. In general, however, an increasing trend can be observed. Our results seem to contradict the prevailing opinion about an increase in the strength of the linkages during the crisis periods. They are also not consistent with the known belief that lower tail dependence becomes stronger in such periods.

Acknowledgments The authors are very grateful to two anonymous referees for their helpful comments and suggestions. Useful comments and remarks from the participants of the 17th International Conference "Forecasting Financial Markets" (May 2010, Hannover, Germany) and the 4th Conference on Computational and Financial Econometrics (December 2010, London, UK) are also much appreciated.

This work was financed from the Polish science budget resources in the years 2010-2013 as the research project N N111 035139. 
Open Access This article is distributed under the terms of the Creative Commons Attribution License which permits any use, distribution, and reproduction in any medium, provided the original author(s) and the source are credited.

\section{References}

Ammer J, Mei J (1996) Measuring international economic linkages with stock market data. J Financ $51: 1743-1763$

Ang A, Bekaert G (2002) International asset allocation with regime shifts. Rev Financ Stud 15:1137-1187

Ang A, Chen J (2002) Asymmetric correlations of equity portfolios. J Financ Econ 63:443-494

Bachman B, Choi JJ, Jeon B, Kopecky K (1996) Common factors in international stock prices: evidence from a cointegration study. Int Rev Financ Anal 5:39-53

Bailey W, Choi JJ (2003) Editors' introduction. International market linkages. J Econ Bus 55:399-404

Bartram S, Taylor S, Wang Y-H (2007) The euro and European financial market dependence. J Bank Financ 31:1461-1481

Beine M, Laurent S (2001) Structural change and long memory in volatility: new evidence from daily exchange rates. In: Dunis C, Timmermann A, Moody J (eds) Developments in forecast combination and portfolio choice. Wiley, Hoboken, pp 159-177

Campbell JY, Hamao Y (1992) Predictable stock returns in the United States and Japan: a study of longterm capital market integration. J Financ 47:43-69

Capiello L, Engle RF, Sheppard K (2006) Asymmetric dynamics in the correlations of global equity and bond returns. J Financ Econom 4:537-572

Chen S, Poon S-H (2007) Modelling international stock market contagion using copula and risk appetite. MBS Working Paper Series, SSRN: http://ssrn.com/abstract=1024288

Chollete L, Heinen A, Valdesogo A (2009) Modeling international financial returns with a multivariate regime switching copula. J Financ Econom 7:437-480

Doornik JA (2006) An object-oriented matrix programming language - Ox ${ }^{\mathrm{TM}}$. Tiberlake Consultants Ltd, London

Embrechts P, McNeil A, Straumann D (2002) Correlation and dependence in risk management: properties and pitfalls. In: Risk management: value at risk and beyond. Cambridge University Press, Cambridge, pp 176-223

Forbes K, Rigobon R (2002) No contagion, only interdependence: measuring stock markets comovements. J Financ 57:2223-2261

Gallo GM, Otranto E (2008) Volatility spillovers, interdependence and comovements: a Markov switching approach. Comput Stat Data Anal 52:3011-3026

Gallo GM, Velucchi M (2009) Market interdependence and financial volatility transmission in East Asia. Int J Financ Econ 14:24-44

Garcia R, Tsafack G (2011) Dependence structure and extreme comovements in international equity and bond markets. J Bank Financ 35:1954-1970

Gérard B, Thanyalakpark K, Batten JA (2003) Are the East Asian markets integrated? Evidence from the ICAPM. J Econ Bus 55:585-607

Hamilton JD (1994) Time series analysis. Princeton University Press, Princeton

Hamao Y, Masulis RW, Ng V (1990) Correlations in price changes and volatility across international stock markets. Rev Financ Stud 3:281-307

Hansen PR, Lunde A (2010) MulCom 2.00, an Ox ${ }^{\mathrm{TM}}$ software package for multiple comparisons. http:// mit.econ.au.dk/vip_htm/alunde/mulcom/mulcom.htm

Hansen PR, Lunde A, Nason JM (2003) Choosing the best volatility models: the model confidence set approach. Oxford B Econ Stat 65:839-861

Hansen PR, Lunde A, Nason JM (2011) The model confidence set. Econometrica 79:453-497

Joe H, Xu JJ (1996) The estimation method of inference functions for margins for multivariate models. Technical Report no 166, Department of Statistics, University of British Columbia

Jondeau E, Rockinger M (2006) The Copula-GARCH model of conditional dependencies: an international stock market application. J Int Money Financ 25:827-853

Karolyi GA, Stulz RM (1996) Why do markets move together? An investigation of U.S.-Japan stock market comovements. J Financ 51:951-986 
Lambert P, Laurent S (2001) Modelling financial time series using GARCH-type models with a skewed Student distribution for the innovations. Institut de Statistique, Université Catholique de Louvain, Discussion Paper 0125

Levy H, Sarnat M (1970) International diversification of investment portfolios. Am Econ Rev 60:668-675 Markwat T, Kole E, van Dijk D (2009) Time variation in asset return dependence: strength or structure? ERIM Report Series 2009-052-F\&A

McNeil AJ, Frey A, Embrechts P (2005) Quantitative risk management. Princeton University Press, Princeton

Nelsen RB (2006) An introduction to copulas. Springer Science+Business Media Inc., New York

Okimoto T (2008) New evidence of asymmetric dependence structures in international equity markets. J Financ Quant Anal 43:787-815

Patton AJ (2004) On the out-of-sample importance of skewness and asymmetric dependence for asset allocation. J Financ Econom 2:130-168

Patton AJ (2006) Modelling asymmetric exchange rate dependence. Int Econ Rev 47:527-556

Patton AJ (2009) Copula-based models for financial time series. In: Andersen TG, Davies RA, Kreiss J-P, Mikosch T (eds) Handbook of financial time series. Springer, Berlin, pp 767-785

Pindick RS, Rotemberg JJ (1993) The comovement of stock prices. Q J Econ 108:1073-1104

Rodriguez JC (2007) Measuring financial contagion: a copula approach. J Empir Financ 14:401-423

Sklar A (1959) Fonctions de répartition à n dimensions et leurs marges. Publ Inst Stat Univ Paris 8:229_ 231

Solnik BH (1974) Why not diversify internationally rather than domestically? Financ Anal J 30:48-54 